\title{
Tibial and fibular nerves evaluation using intraoperative electromyography in rats $^{1}$
}

\author{
André Coelho NepomucenoI, Elisa Landucci PolitaniII, Eduardo Guandelini da Silva ${ }^{\mathrm{II}}$, Raquel Salomone ${ }^{\mathrm{III}}$, Marco Vinicius \\ Losso Longo ${ }^{I v}$, Alessandra Grassi Sallesv, José Carlos Marques de Faria ${ }^{\text {VI, Rolf Gemperli }}{ }^{\mathrm{VI}}$
}

DOI: http://dx.doi.org/10.1590/S0102-865020160080000007

IMD, Plastic Surgeon, Fellow PhD degree, General Surgery Program, Department of Plastic Surgery and Microsurgery, Medical School, Universidade de São Paulo (USP), Brazil. Design the protocol, scientific content of the study, technical procedures, manuscript preparation and writing, English language.

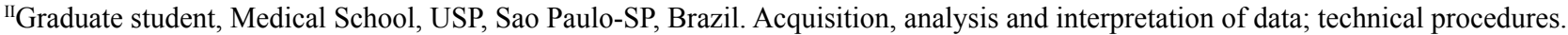

IIIPD, Otorhinolaryngologist, Department of Otorhinolaryngology, USP, Sao Paulo-SP, Brazil. Design the protocol; technical procedures; acquisition, analysis and interpretation of data; manuscript writing; critical revision.

${ }^{\text {IV }} \mathrm{PhD}$, Plastic Surgeon, Department of Plastic Surgery and Microsurgery, Medical School, USP, Sao Paulo-SP, Brazil. Technical procedures, analysis of data.

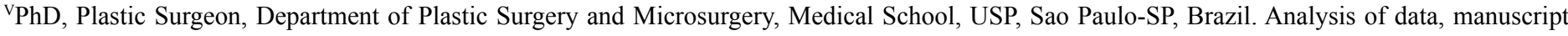
writing, critical revision.

${ }^{\mathrm{V}}$ Full Professor, Plastic Surgeon, Department of Plastic Surgery and Microsurgery, Medical School, USP, Sao Paulo-SP, Brazil. Intellectual and scientific content of the study, critical revision.

${ }^{\mathrm{VII}}$ Chairman and Head, Department of Plastic Surgery and Microsurgery, Medical School, USP, Sao Paulo-SP, Brazil. Intellectual and scientific content of the study.

\section{ABSTRACT}

PURPOSE: To evaluate a new model of intraoperative electromyographic (EMG) assessment of the tibial and fibular nerves, and its respectives motor units in rats.

METHODS: Eight Wistar rats underwent intraoperative EMG on both hind limbs at two different moments: week 0 and week 12. Supramaximal electrical stimulation applied on sciatic nerve, and compound muscle action potential recorded on the gastrocnemius muscle (GM) and the extensor digitorum longus muscle (EDLM) through electrodes at specifics points. Motor function assessment was performaced through Walking Track Test.

RESULTS: Exposing the muscles and nerves for examination did not alter tibial $(p=0.918)$ or fibular $(p=0.877)$ function between the evaluation moments. Electromyography of the GM, innervated by the tibial nerve, revealed similar amplitude ( $p=0.069)$ and latency $(\mathrm{p}=0.256)$ at week 0 and at 12 weeks, creating a standard of normality. Meanwhile, electromyography of the EDLM, innervated by the fibular nerve, showed significant differences between the amplitudes $(p=0.003)$ and latencies $(p=0.021)$ at the two different moments of observation.

CONCLUSION: Intraoperative electromyography determined and quantified gastrocnemius muscle motor unit integrity, innervated by tibial nerve. Although this study was not useful to, objectively, assess extensor digitorum longus muscle motor unit, innervated by fibular nerve.

Key words: Electromyography. Tibial Nerve. Peroneal Nerve. Rats, Wistar. 


\section{Introduction}

A variety of experimental models have been used to study processes involving degeneration and regeneration of the peripheral nerves; animal models such as dogs and cats ${ }^{1}$, rabbits $^{2}$, guinea pigs ${ }^{3}$, pigs ${ }^{4}$, and rats $^{5,6}$ have been used, each with its own advantages and disadvantages, but we still remain far from the ideal model.

One well-established model is the sciatic nerve in rats ${ }^{7}$. However, questions related to surgical treatment of facial paralysis have led to double muscle innervation hypothesis ${ }^{8}$. Therefore, to enlighten this issue, two different motor units experimental model become interesting, making the study of the tibial and fibular branches of the sciatic nerve relevant.

The goal of electrophysiological studies is to determine and quantify the function and disorders of the peripheral nervous system, specifically of the motor nerves and motor unit ${ }^{9}$, becoming a fundamental tool on nerve regeneration assessment. Factors such as anatomy, electrode type and distance, location and intensity of electrical stimulation, and surgical fibrosis could affect results ${ }^{10}$. The aim of this study was to evaluate a new model of intraoperative electromyographic assessment of the tibial nerve, fibular nerve, and its respectives motor units in experimental studies with rats.

In the technique proposed here, supramaximal electrical stimulation directly applied onto isolated sciatic nerve, through a bipolar needle electrode, at a fixed point $10 \mathrm{~mm}$ proximal to its trifurcation into the tibial, fibular, and sural branches, was recorded into gastrocnemius muscle (GM) and extensor digitorum longus muscle (EDLM).

\section{Methods}

This study was approved by Universidade de São Paulo (USP) research ethical committee under process number 157/13.

In sample size calculation, the following assumptions were accepted: data are paired and come from the same population; each pair is chosen randomly and independently; minimal number of animals for $p<0.05$ and equal coefficient of variance due to biological variability. Normality assumption was rejected and it was used a nonparametric test, the Fligner-Policello modification of the Mann-Whitney-Wilcoxon, to determine the sample size ${ }^{11}$. Therefore, 8 adult male Wistar rats weighing between 300-350 g were placed under general anesthesia via intraperitoneal injection of pentobarbital sodium at a dose of $30-45 \mathrm{mg} / \mathrm{kg}^{12}$. The animals were positioned in ventral decubitus, right and left hind limbs were shaved and cleaned with a polyvinylpyrrolidone $10 \%$ solution.
A $3 \mathrm{~cm}$ skin incision was made longitudinally on the posterior aspect of each thigh, from the greater trochanter to the knee. Blunt dissection was performed between the gluteus maximus and biceps femoris muscles, and sciatic, tibial, fibular, and sural nerves were exposed, along with the gastrocnemius muscle (GM). After intraoperative electromyography (EMG) was performed, the incisions were closed along the muscular planes and the skin using 6.0 nylon suture.

After 12 weeks, once again, all rats underwent new intraoperative EMG, followed by euthanasia with an intraperitoneal injection of pentobarbital sodium $(100 \mathrm{mg} / \mathrm{kg})^{12}$.

\section{Functional assessment}

Walking track analysis was carried out prior to any other procedure, to calculate the baseline values for the tibial functional index (TFI) and fibular functional index (FFI) for both hind limb, and was repeated after 12 weeks ${ }^{13}$. For gait analysis, the following characteristics were measured: print length (PL), print width (PW), and intermediary toe-spread distance (ITSD) in both the experimental (E) and the normal side $(\mathrm{N})$. As suggested by Bain (1989), the tibial nerve functional index (TFI) was calculated using the formula: $T F I=-37.2 \times[(P L E-P L N) /(P L N)]+104.4$ $x[(P W E-P W N) / P W N]+45.6 x[(I T S D E-I T S D N) / I T S D N)]$ - 8.8. The FFI was calculated using the formula: $F F I=174.9 x$ $[(P L E-P L N) /(P L N)]+80.3 x[(P W E-P W N) / P W N]-13.4$.

\section{Electrophysiological evaluation}

Intraoperative electrophysiologic testing was conducted in both hind limb at week 0 and after 12 weeks.

During the surgical procedure, the animals had the body temperature assessed through a rectal thermometer and kept around $34^{\circ} \mathrm{C}$, and the ambient temperature was maintained around $25^{\circ} \mathrm{C}$. The sciatic nerve was then stimulated and the compound muscle action potential (CMAP) was recorded in the compartments of the tibial and fibular nerves on the side ipsilateral to the stimulation. The CMAP parameters analyzed were amplitude and latency.

The CMAP was obtained using a Neuro-MEP-Micro model portable EMG device (Neurosoft $^{\circledR}$, Ivanovo, Russia) connected to a Hewlett-Packard Pavilion laptop (HewlettPackard $^{\circledR}$, Palo Alto, CA, USA) by a universal serial bus connector (USB), eliminating the need for an external power source. NeuroMEP.NET software version 2.4.23.0 was used to configure the EMG device (high-pass $10 \mathrm{~Hz}$ filter; low-pass $10 \mathrm{kHz}$ filter; notch filter off; signal input margin of $60 \mathrm{mV} ; 10 \mathrm{kHz}$ sampling rate) and 
to visualize and analyze the CMAPs.

For electrical stimulation and recording, two monopolar $12 \mathrm{~mm}$ length and $0.35 \mathrm{~mm}$ diameter subdermal teflon needle electrodes (Spes Medica ${ }^{\circledR}$, São Paulo, Brazil) were used, arranged in parallel at a fixed distance of $5 \mathrm{~mm}$ from each other. The tips of the stimulus electrodes were curved beforehand for better fit and to prevent injuries (Figure 1A). The recording electrodes had an insulating coating over $9 \mathrm{~mm}$ of their length, leaving the distal $3 \mathrm{~mm}$ uncoated (Figure 1B). A monopolar electrode of the same brand with a similar coating to the electrodes described above was used as a ground (neutral), but was positioned at the midpoint between the stimulation and the recording electrodes. If impedance exceeded five $\Omega$, the electrodes were relocated or replaced.
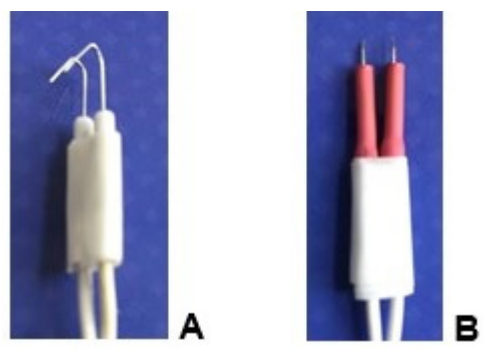

FIGURE 1 - Electrode model used for electrical stimulation (A). Electrode model used for electrical recording (B).

The stimulus electrodes were located in the sciatic nerve, $10 \mathrm{~mm}$ proximal to its trifurcation (Figure 2), anode $(+)$ was placed proximally and the cathode (-) distally from this point. The electrical stimuli used were unique, without promeasurement, and had a duration of $0.2 \mathrm{~ms}$. Initial intensity was $0.1 \mathrm{~mA}$ and gradually raised until supramaximum stimulus was reached.

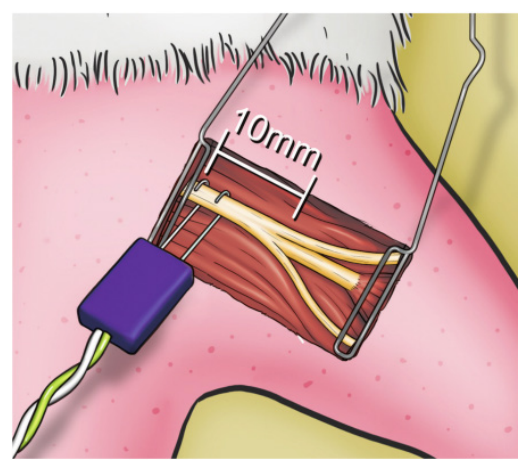

FIGURE 2 - Stimulus electrode on sciatic nerve, positioned 10 $\mathrm{mm}$ proximally to its trifurcation.

In the tibial nerve compartment, represented by the GM, the CMAP was recorded through the distal part of the electrode inserted into the belly of the GM at its midpoint between the proximal portion and its distal tendon, at a distance of $15 \mathrm{~mm}$ longitudinally from the trifurcation of the sciatic nerve (Figure 3), parallel to the fibers and ipsilateral to the stimulated side, with the proximal recording pole and the reference pole more distal in relation to the stimulator. The scan used was $1.0 \mathrm{~ms} /$ division with a total window of $10 \mathrm{~ms}$ and a gain of $2.5 \mathrm{mV} /$ division.

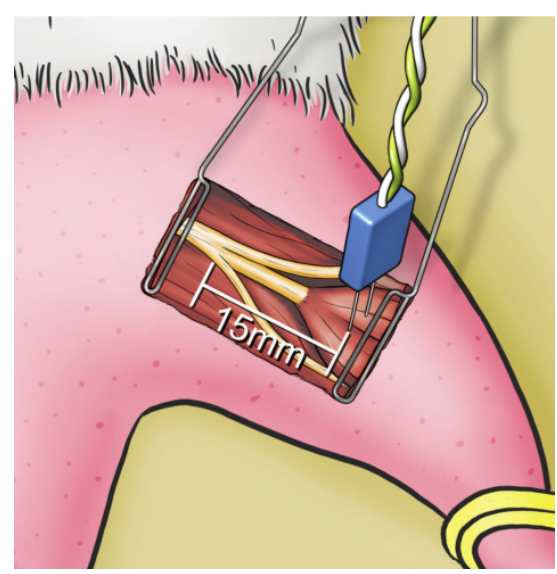

FIGURE 3 - Recording electrode into gastrocnemius muscle (tibial nerve compartment), positioned $15 \mathrm{~mm}$ distally from sciatic nerve trifurcation.

In the fibular nerve compartment, represented by extensor digitorum longus muscle (EDLM), CMAP was recorded through the distal part of the electrode inserted longitudinally into the belly of the EDLM, $20 \mathrm{~mm}$ from the rat's knee joint, via a percutaneous introduction (Figure 4), parallel to the fibers and ipsilateral to the stimulated side, with the proximal recording pole and the reference pole more distal in relation to the stimulator. The scan used was $1.0 \mathrm{~ms}$ /division with a total window of $10 \mathrm{~ms}$ and a gain of 2.5 $\mathrm{mV} /$ division.

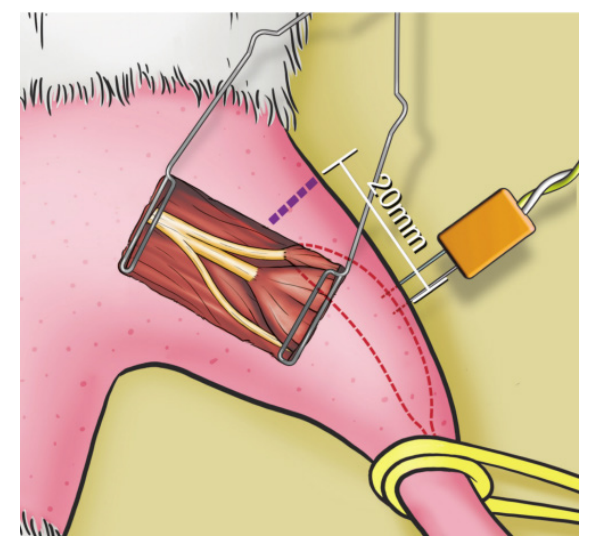

FIGURE 4 - Recording electrode into extensor digitorum longus muscle (fibular nerve compartment), positioned $20 \mathrm{~mm}$ distally from the knee joint. 
The electrodes positioned together for the intraoperative EMG are shown in Figure 5.

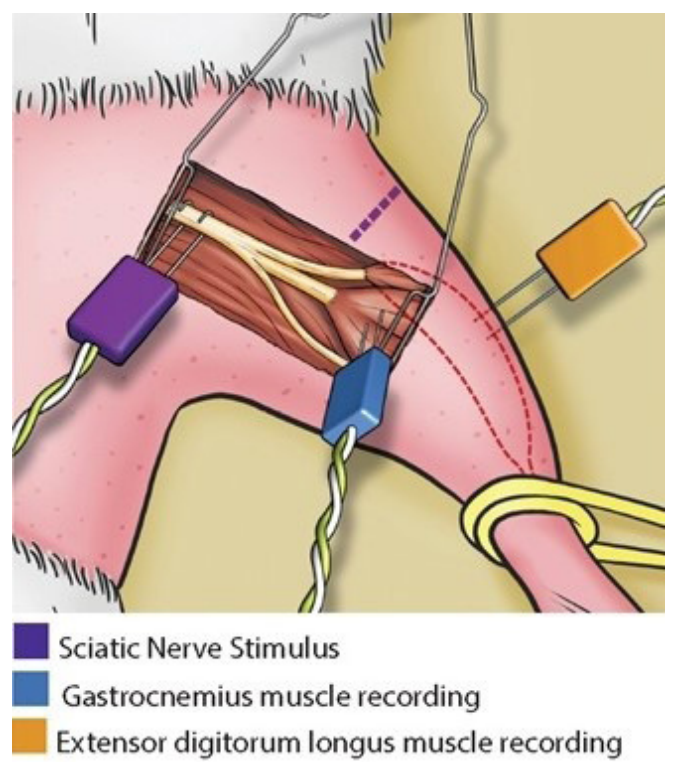

FIGURE 5 - Intraoperative electromyography: stimulus in the sciatic nerve, recording in the gastrocnemius muscle (tibial nerve compartment) and recording in the extensor digitorum longus muscle (fibular nerve compartment).

The postoperative analgesia was done with Buprenorfin, $0.05 \mathrm{mg} / \mathrm{Kg}$, subcutaneously, every 12 hours for five days ${ }^{12}$.

\section{Statistical analysis}

The results of the tests were expressed as means and standard deviations and compared using Statistical Package for Social Sciences (SPSS) software version 19.0, specifically through the nonparametric Wilcoxon signed-rank test, with the level of significance set at $5 \%(\mathrm{p}<0.05)$.

\section{Results}

\section{Functional assessment}

The TFI for the right and left hind limbs of the 8 animals revealed statistical similarity in both week $0(\mathrm{p}=0.208)$ and week $12(p=0.263)$. Similarly, the FFI for the animals' right and left hind limbs revealed statistical similarity in both week $0(\mathrm{p}=0.074)$ and week $12(p=0.735)$.

Since there was no statistically significant difference between the sides for the evaluated parameters, we can consider the values for both sides in sets of 16 hind limbs and represent them by their means, generating new values and obtaining a normal pattern, since the values for the 16 TFIs in week 0 and week 12 were similar ( $\mathrm{p}=0.918$ ), as the FFIs in week 0 and week 12 were also similar $(\mathrm{p}=0.877)$.

\section{Electrophysiological evaluation}

As for the tibial nerve and the GM, the amplitude of the right and left hind limbs of the 8 animals revealed statistical similarity in both week $0(\mathrm{p}=0.999)$ and week $12(\mathrm{p}=0.069)$. Latency also was seen to be similar between both sides in both week $0(p=0.270)$ and week $12(p=0.575)$.

With regard to the fibular nerve and the EDLM, the amplitude of the right and left hind limbs of the 8 animals revealed statistical similarity in both week $0(p=0.575)$ and week 12 $(\mathrm{p}=0.779)$. As well as latency in both week $0(\mathrm{p}=0.528)$ and week $12(p=0.325)$ between both sides.

Since there was no statistically significant difference between the sides for the parameters evaluated, we can consider the values for both sides in sets of 16 hind limbs and represent them by their means, generating new EMG values in week 0 and week 12. Thus, comparing the electromyography (EMG) outcomes of the 16 hind limbs, between the two assessment moments, it was revealed that GM amplitude and latency were similar, while EDLM amplitude and latency were statistically different (Table 1).

TABLE 1 - Comparison between the two moments of evaluation in terms of gastrocnemius muscle (GM) and extensor digitorum longus muscle (EDLM) amplitude and latency.

\begin{tabular}{cccc}
\hline EMG & $\mathrm{n}$ & Mean $(\mathrm{SD})$ & $\mathrm{p}$ value \\
\hline Amplitude GM 0 & 16 & $4.22( \pm 4.68) \mathrm{mV}$ & \\
Amplitude GM 12 & 16 & $7.49( \pm 6.24) \mathrm{mV}$ & 0.069
\end{tabular}

\begin{tabular}{cccc} 
Amplitude EDLM 0 & 16 & $3.65( \pm 3.15) \mathrm{mV}$ & $0.003^{*}$ \\
Amplitude EDLM 12 & 16 & $7.67( \pm 3.81) \mathrm{mV}$ & \\
Latency GM 0 & 16 & $1.56( \pm 0.56) \mathrm{ms}$ & 0.256 \\
Latency GM 12 & 16 & $2.44( \pm 3.31) \mathrm{ms}$ & \\
Latency EDLM 0 & 16 & $1.44( \pm 0.35) \mathrm{ms}$ & $0.021^{*}$ \\
Latency EDLM 12 & 16 & $1.66( \pm 0.27) \mathrm{ms}$ & \\
\hline
\end{tabular}

* Statistically significant

\section{Discussion}

Small animals like rats, especially the Wistar breed, are widely used in experimental research because they are 
easily handled, highly resistant to manipulation and infections, and inexpensive ${ }^{14}$; additionally, all their metabolic and growth parameters have been well defined and described in the literature ${ }^{15}$.

Because it is quite long and and easily exposed, the sciatic nerve of Wistar rats is one of the most commonly used models in studies investigating neural regeneration ${ }^{16}$. However, Kemp ${ }^{17}$ showed that reinnervation occurs after nervous transfer between the tibial and fibular nerves, revealing that these branches of the sciatic nerve may be used in experimental studies of peripheral nerves. However, inconsistent outcomes may be found if analysis method is not standardized ${ }^{5}$.

In peripheral nerve regeneration study the assessment moment is crucial. It was demonstrated that 12 weeks after nerve repair, axonal count reaches its maximum ${ }^{18}$. English ${ }^{19}$ showed that recovery of electromyographic activity after transection and surgical repair of the sciatic nerve in rats happens gradually, reaching highest values from 8 weeks after the experiment.

This study revealed that invasive surgical exposure of the sciatic nerve and its branches, associated with intraoperative electromyography did not lead to gait functional changes 12 weeks after the first procedure.

Rupp $^{20}$ stimulated sciatic nerve and recorded CMAP in GM, with a concentric needle, in terms of nerve conduction velocity, 8 weeks after the experiment. The concentric needle electrode was seen to capture smaller CMAPs, or none at all, compared to monopolar electrodes. This study also reported that high intensity stimuli (>1.9 mA) generated CMAPs in the GM, even after injury without repair of sciatic nerve, suggesting that the impulses may have been propagated by contiguous muscles. This may corroborate to inconsistents EMG outcomes in sciatic nerve experimental studies, showing that the adjacent muscles have a strong influence on recording from the GM and vice versa.

Despite higher readings in week 12, compared with week 0 , there was no significant statistical difference between these two moments regarding GM amplitude $(\mathrm{p}=0.069)$ and latency $(p=0.256)$. These findings suggests that intraoperative EMG in moment zero could be used as a normal control, dismissing the need for a sham group just for EMG in a later moment of observation.

On fibular nerve compartment, there was a significant statistical difference between the two evaluation moments in terms amplitude $(p=0.003)$ and latency $(p=0.021)$. The EDLM is located in the anterior compartment of the leg and is substantially less bulky than the GM. To avoid increased morbidity in the rat's leg, as result of unnecessary dissection, the recording electrode was percutaneously introduced at a fixed point $2 \mathrm{~cm}$ distal from the knee joint. Surgical fibrosis and cross reading contamination from adjacent muscles may be the reason for these changes at the different times ${ }^{21}$.

Stipp-Brambilla ${ }^{22}$ compared data between percutaneous and intraoperative EMG. The tibial nerve was sectioned, so there was no interference from the GM in the reading for the fibular compartment. Stimulus was applied in the sciatic nerve using a hooked bipolar electrode at a frequency of 1 pps and a duration of $100 \mu \mathrm{s}$. CMAP was recorded in the cranial tibial muscle. These authors concluded that surgical exposure did not significantly affect the values observed for the electrophysiological parameters, and even made it possible to stimulate an exact point in the target nerve, but did not specify this point in the sciatic nerve nether the recording location in the cranial tibial muscle. Cranial tibial muscle has a greater volume than the EDLM, the target muscle for the fibular nerve compartment used in our study. This fact may justify the higher amplitude and area values found by these authors ${ }^{23}$

Egeland $^{24}$ sectioned tibial and sural nerves, then stimulated the sciatic nerve with $0.2 \mathrm{~mA}( \pm 0.14)$ and obtained a CMAP recording in the EDLM (the target muscle of the fibular nerve) with an amplitude of $16.63 \mathrm{mV}( \pm 0.5 .20)$, latency of 1.34 $\mathrm{ms}( \pm 0.23)$, and nerve conduction velocity of $22.19 \mathrm{~m} / \mathrm{s}( \pm 3.62)$. This author repeated the study after sectioning the tibial and fibular nerves, and noted the transmission of a discrete antidromic action potential (afferent) in the sural nerve (sensitive). Therefore concluded that the presence of the intact sural nerve could lead to contamination recording in neurophysiological assessment of the tibial and fibular branches. This suggests future studies, that intraoperatory section of non target branches would avoid contaminating responses in neurophysiological assessment of the target nerve.

Tatlidede $^{25}$ proposed an experimental model using lateral tibial nerve and the flexor digiti minimi brevis (FDMB) muscle. Stimulus was applied through a bipolar hook-shaped electrode in distal third of the leg, and CMAP recorded through a concentric needle electrode. Control group, without nerve injury, outcomes revealed a $1 \mathrm{~mA}$ stimulus in the tibial nerve yielded a 1 $\mathrm{mV}$ amplitude in the FDMB after 12 weeks. Despite similarities to our study with regard to experiment duration and electrodes, this author did not establish distance parameters for stimulus and recording points; additionally, the FDMB is a small muscle and is more subject to contamination from recording of CMAPs in the adjacent muscles ${ }^{20}$.

Different distances between stimulus and recording points could change parameters such amplitude and latency, 
making the experimental model difficult to reproduce ${ }^{10}$.

Another important EMG standardization variable is the intensity of stimulus current. Amplitude exhibits a direct relationship with the number of nerve fibers depolarized by the stimulus. A lower amplitude may reflect a decrease in the number of fibers or decrease in the synchrony of the response. To avoid errors in assessing amplitude, the supramaximal stimulus, which is the maximum intensity of stimulus required to depolarize all fibers, should be used ${ }^{26}$.

Several of the studies described above also used a wide variation in stimulus intensity, so the responses obtained may not reflect supramaximal amplitude.

EMG is a useful and objective method for evaluating nerve regeneration. The use of supramaximal stimulus and standardization of electrode distances in relation to reference points, as well as the use of amplitude and latency, as more relevant variables, could assist when comparing EMG outcomes in future experimental studies involving nerve regeneration of the tibial and fibular nerves in rats.

In a future study, the model presented for intraoperative EMG evaluation will be used to assess progress after injury and different techniques of nerve repair, including double innervation.

\section{Conclusions}

Intraoperative electromyography determined and quantified gastrocnemius muscle motor unit integrity, innervated by tibial nerve. Although this study was not useful to, objectively, assess extensor digitorum longus muscle motor unit, innervated by fibular nerve. The methodology described allows the tibial or fibular nerve to be analyzed separately, sectioning the nerve that is not relevant at the evaluation moment.

\section{References}

1. van Soens I, Struys MM, van Ham LM. Muscle potentials evoked by magnetic stimulation of the sciatic nerve in unilateral sciatic nerve dysfunction. J Small Anim Pract. 2010;51(5):275-9. PMID: 20214726

2. Reichert P, Kiełbowicz Z, DziEgiel P, Puła B, Kuryszko J, Gosk $\mathrm{J}$, Bocheńska A. The rabbit brachial plexus as a model for nerve repair surgery - histomorphometric analysis. Anat Rec (Hoboken). 2015;298(2):444-54. PMID: 25284580.

3. Aguiar J, Mogridge G, Hall J. Femoral fracture repair and sciatic and femoral nerve blocks in a guinea pig. J Small Anim Pract. 2014;55(12):635-9.PMID: 23398458.

4. Belda E, Laredo FG, Gil F, Soler M, Murciano J, Ayala MD, Gómez S, Castells MT, Escobar M, Agut A. Ultrasound-guided administration of lidocaine into the sciatic nerve in a porcine model: correlation between the ultrasonographic evolution of the lesions, locomotor function and histological findings. Vet J. 2014;200(1):170-4. PMID: 24594382.

5. Wood MD, Kemp SW, Weber C, Borschel GH, Gordon $\mathrm{T}$. Outcome measures of peripheral nerve regeneration. Ann Anat. 2011;193(4):321-33. PMID: 21640570.

6. Salomone R, Bento RF, Costa HJ, Azzi-Nogueira D, Ovando PC, Da-Silva CF, Zanatta DB, Strauss BE, Haddad LA. Bone marrow stem cells in facial nerve regeneration from isolated stumps. Muscle Nerve. 2013;48(3):423-9. PMID: 23824709.

7. Savastano LE, Laurito SR, Fitt MR, Rasmussen JA, Gonzalez Polo V, Patterson SI. Sciatic nerve injury: a simple and subtle model for investigating many aspects of nervous system damage and recovery. J Neurosci Methods. 2014;227:166-80. PMID: 24487015.

8. Cardenas-Mejia A, Covarrubias-Ramirez JV, Bello-Margolis A, Rozen S. Double innervated free functional muscle transfer for facial reanimation. J Plast Surg Hand Surg. 2015;49(3):183-8. PMID: 25469588.

9. Robinson AJ, Snyder-Mackler L. Eletrofisiologia clínica: eletroterapia e teste eletrofisiológico. 2ed. Porto Alegre: Artmed Editora; 2001.

10. Salomone R, Costa HJZR, Rodrigues JRF, Silva SMRE, Ovando, Bento RF. Assessment of a neurophysiological model of the mandibular branch of the facial nerve in rats by electromyography. Ann Otol Rhinol Laryng. 2012;121:179-84. PMID: 25469588.

11. Scheibe PO. Number of samples-hypothesis testing. Nucl Med Biol. 2008;35:3-9. PMID: 18158937.

12. Damy SB, Camargo RS, Chammas R, Figueiredo LFP. Aspectos fundamentais da experimentação animal - aplicações em cirurgia experimental. Rev Assoc Med Bras. 2010;56(1):103-11. doi: 10.1590/S0104-42302010000100024.

13. Bain JR, Mackinnon SE, Hunter DA. Functional evaluation of complete sciatic, peroneal, and posterior tibial nerve lesions in the rat. Plast Reconstr Surg. 1989;83(1):129-38. PMID: 2909054.

14. Mattox DE, Felix H. Surgical anatomy of the rat facial nerve. Am J Otol. 1987;8(1):43-7. PMID: 3565547.

15. Donaldson HH. The Rat. Reference tables and data for the albino rat (Mus norvegicus albinus) and the Norway rat (Mus norvegicus). 2ed. Memoirs No 6. Wistar Inst. Anat Biol..Philadelphia; 1924.

16. Geuna $\mathrm{S}$. The sciatic nerve injury model in pre-clinical research. J Neurosci Methods. 2015;243:39-46. PMID: 25629799.

17. Kemp SW, Alant J, Walsh SK, Webb AA, Midha R. Behavioural and anatomical analysis of selective tibial nerve branch transfer to the deep peroneal nerve in the rat. Eur J Neurosci. 2010;31(6):1074-90. PMID: 20377620.

18. Mackinnon SE, Dellon AL, O'Brien JP. Changes in nerve fiber numbers distal to a nerve repair in the rat sciatic nerve model. Muscle Nerve. 1991;14(11):1116-22. PMID: 1745287.

19. English AW, Chen Y, Carp JS, Wolpaw JR, Chen XY. Recovery of electromyographic activity after transection and surgical repair of the rat sciatic nerve. J Neurophysiol. 2007;97(2):1127-34. PMID: 17122310 .

20. Rupp A, Dornseifer U, Fischer A, Schmahl W, Rodenacker K, Jütting U, Gais P, Biemer E, Papadopulos N, Matiasek K. Electrophysiologic assessment of sciatic nerve regeneration in the rat: surrounding limb muscles feature strongly in recordings from the gastrocnemius muscle. J Neurosci Methods. 2007;166(2):26677. PMID: 17854904.

21. English AW, Weeks OI. Electromyographic cross-talk within a compartmentalized muscle of the cat. J Physiol. 1989;416:327-36. PMID: 2558175 .

22. Stipp-Brambilla EJ, Romão AM, Garbino JA, Salgado MH, Viterbo F. Efeito da exposição cirúrgica de nervos e músculos no teste neurofisiológico em ratos. Acta Fisiatr. 2010;17(3):109-111. 
23. Wolthers M, Moldovan M, Binderup T, Schmalbruch H, Krarup C. Comparative electrophysiological, functional, and histological studies of nerve lesions in rats. Microsurgery. 2005;25:508-19. PMID: 16145683.

24. Egeland BM, Urbanchek MG, Peramo A, Richardson-Burns SM, Martin DC, Kipke DR, Kuzon WM Jr, Cederna PS. In vivo electrical conductivity across critical nerve gaps using poly $(3,4-$ ethylenedioxythiophene)-coated neural interfaces. Plast Reconstr Surg. 2010;126(6):1865-73. PMID: 20700080.

25. Tatlidede S, Gonen E, Baslo B, Soydan T, Solakoglu S, Bas L. A new nerve-muscle unit model for peripheral nerve studies in rats. $\mathrm{J}$ Reconstr Microsurg. 2006;22(7):533-8. PMID: 17048136.

26. Hayashi A, Yanai A, Komuro Y, Nishida M, Inoue M, Seki T. Collateral sprouting occurs following end-to-side neurorraphy. Plast Reconstr Surg. 2004;114 (1):129-37. PMID: 15220580.

\section{Correspondence:}

André Coelho Nepomuceno

Avenida Dr. Arnaldo, 255/sala 1363

01246-903 São Paulo - SP Brasil

Tel.: (55 11)3062-0415

andreconep@yahoo.com.br

Received: Apr 18, 2016

Review: Jun 20, 2016

Accepted: July 21, 2016

Conflict of interest: none

Financial source: CAPES

${ }^{1}$ Research performed at Department of Plastic Surgery and Microsurgery, Medical School, Universidade de São Paulo (USP), Brazil. 